\title{
The action of sennosides and related compounds on human colon and rectum ${ }^{1}$
}

\author{
J. D. HARDCASTLE AND J. L. WILKINS \\ From the London Hospital and St Mark's Hospital, London
}

SUMMARY The direct action of intraluminal senna and related compounds on the human colon and rectum has been investigated. Motility was recorded by balloon kymography with recording units inserted into well established transverse colostomies or into the rectum.

The motility of the colon was not changed by intraluminal senna glycosides but the introduction of senna previously incubated with faeces or Esch. coli stimulated the colon to peristalt. The peristalsis was similar to that stimulated by rheinanthrone, an oxanthrone produced by chemical hydrolysis and reduction of senna.

Both activated senna and rheinanthrone appeared to act in the colon by contact stimulation. No peristaltic response was stimulated in the rectum, either with activated senna or with rheinanthrone.

Concentrated senna extract contains anthracene derivatives which exist mainly in the form of glycosides (sennosides) (Kussmaul and Becker, 1947; Fairbairn, 1949). When hydrolysed these glycosides are converted into aglycones of three main types, anthroquinones, anthranols, and oxanthrones.

Sennosides are many times more effective in producing defaecation when given orally than are the sugar-free aglycones (Fairbairn, 1949), although there is evidence in animals that when directly introduced into the colon, aglycones almost immediately stimulate activity, whilst sennosides are inactive (Okada, 1940). To account for this, it has been postulated that the sugar of the sennosides protects the aglycone moiety from chemical breakdown during its passage through the small bowel (Fairbairn, 1949).

Two possible modes of action of oral senna have been suggested: first that the sennosides are broken down in the small bowel and aglycone derivatives absorbed into the blood stream with

1 This work has been carried out by J. L. Wilkins as part of the requirement of the degree of M.Chir of the University of Cambridge.

Received for publication 4 August 1970. subsequent action on the colon (Straub and Triendl, 1937; Illingworth, 1953), and secondly that most of the sennosides pass unchanged through the small intestine into the colon, where they are changed by bacterial action into the active free aglycones (Okada, 1940; Schmidt, 1960).

In this paper the effects of senna concentrateand of a pure oxanthrone (rheinanthrone) have been investigated in the human colon and rectum, and the mechanism of the action has been studied.

\section{Method}

Intraluminal pressures within the colon and rectum of human subjects have been recorded using small air-filled latex balloons $(0.5 \mathrm{~cm} \times$ $0.8 \mathrm{~cm}$ ) placed $5 \mathrm{~cm}$ apart, connected by fine polythene tubing to transducers and a direct writing recorder (Hardcastle and Mann, 1968). The units were introduced into the colon and rectum through a sigmoidoscope, care being taken to distend the lumen as little as possible. 
Access was gained to the right and left sides of the colon through well established colostomies, the units being placed as far from the opening as possible. After introduction of the units the bowel was left undisturbed for 20 minutes before a study was commenced.

A 30-minute control period of observation was undertaken. The substance under investigation was then introduced into the lumen of the bowel and a further 60-minute observation period performed. If during this time a repetitive peristaltic wave, similar in character to peristaltic activity already described in the colon of man (Hardcastle and Mann, 1968) occurred, the response was considered positive. If spontaneous peristaltic activity was observed in the control period, the study was discontinued as a nonspecific response could then have affected the result (Hardcastle and Mann, 1970).

The senna concentrate that has been used was composed of powdered deseeded pods of Cassia acutifolia containing approximately $45 \mathrm{mg}$ of sennosides A and B per gram and $0.5 \mathrm{~g}$ of this powder was dissolved in saline or thioglycollate broth (Brewer's medium) (Brewer, 1940) before being introduced into the lumen of the bowel.

The effect of previous incubation of senna concentrate with faeces was investigated by obtaining $10 \mathrm{~g}$ of faeces from the proximal stoma of the transverse colostomy and suspending in Brewer's medium. This was divided into two portions; $0.5 \mathrm{~g}$ of senna concentrate was added to one specimen and both were incubated at $38^{\circ} \mathrm{C}$ for six to 12 hours. The control and senna incubated specimens were then tested on the colostomy of the same patient.

The effect of incubation of senna concentrate with Esch. coli was investigated by subculturing the bacterium from a specimen of the patient's faeces; this was then introduced into Brewer's medium and incubated with $0.5 \mathrm{~g}$ of senna concentrate for three hours.

The aglycone compound studied was rheinanthrone produced by chemical hydrolysis and subsequent reduction of senna concentrate.

In the studies in which the effect of a mucosal local anaesthetic was investigated, $2 \mathrm{ml}$ of $4 \%$ lignocaine was introduced into the lumen of the bowel.

\section{Results}

COLON

Detailed analysis of the results is shown in the Table. In six studies senna concentrate dissolved in Brewer's medium did not stimulate peristalsis (Fig. 1); however, a similar mixture previously incubated with faeces at $38^{\circ} \mathrm{C}$ induced peristaltic activity in seven out of eight patients (Fig. 2). A similar effect was seen using pure $E$. coli cultures in place of the faeces (Fig. 3).
Incubation of the senna and faeces in saline was not as effective in stimulating peristalsis,

\begin{tabular}{lll}
\hline Test & $\begin{array}{l}\text { No. of Patients } \\
\text { Studied }\end{array}$ & $\begin{array}{l}\text { Peristalsis } \\
\text { Observed }\end{array}$ \\
\hline $\begin{array}{l}\text { Senna incubated with Brewer's } \\
\text { medium }\end{array}$ & 6 & 0 \\
$\begin{array}{l}\text { Senna incubated with faeces } \\
\text { in Brewer's medium }\end{array}$ & 8 & 7 \\
$\begin{array}{l}\text { Senna incubated with faeces } \\
\text { in saline }\end{array}$ & 6 & 3 \\
$\begin{array}{l}\text { Senna incubated with } \text { E. coli } \\
\text { in Brewer's medium }\end{array}$ & 3 & 3 \\
$\begin{array}{l}\text { Rheinanthrone suspended } \\
\text { in Brewer's medium }\end{array}$ & 7 & 6 \\
$\begin{array}{l}\text { Faeces incubated in Brewer's } \\
\text { medium }\end{array}$ & 4 & 0 \\
\hline
\end{tabular}

Table Analysis of the peristalsis observed after the six studies

only three of the six studies being positive. Rheinanthrone suspended in Brewer's medium stimulated peristalsis in six out of seven patients (Fig. 4). In four control studies faeces incubated with Brewer's medium did not alter colonic motility.

\section{RECTUM}

In seven studies senna concentrate incubated in Brewer's medium did not affect rectal activity. In four patients senna and faeces incubated together in Brewer's medium did not stimulate peristalsis but a rise in the baseline of the trace was noticed on one occasion. In six studies rheinanthrone suspended in Brewer's medium did not stimulate peristalsis; a rise in the baseline was noticed in two of the patients. In three studies in which faeces previously incubated with Brewer's medium was introduced into the rectum no change in rectal motility was observed.

EFFECT OF LOCAL ANAESTHETIC ON THE COLONIC PERISTALTIC RESPONSE

In four patients the previous application of $2 \mathrm{ml}$ of $4 \%$ lignocaine to the colonic mucosa blocked the peristaltic response to the subsequent introduction of both rheinanthrone and senna incubated with faeces (Fig. 5) and in a further study the response was partially blocked.

In four studies the introduction of lignocaine into the bowel after a peristaltic response had been established did not alter the character or the rate of progression of the repetitive peristaltic response (Fig. 6). The frequency of the response was, however, reduced.

\section{Discussion}

In this study senna concentrate introduced into 
Senna Conc.
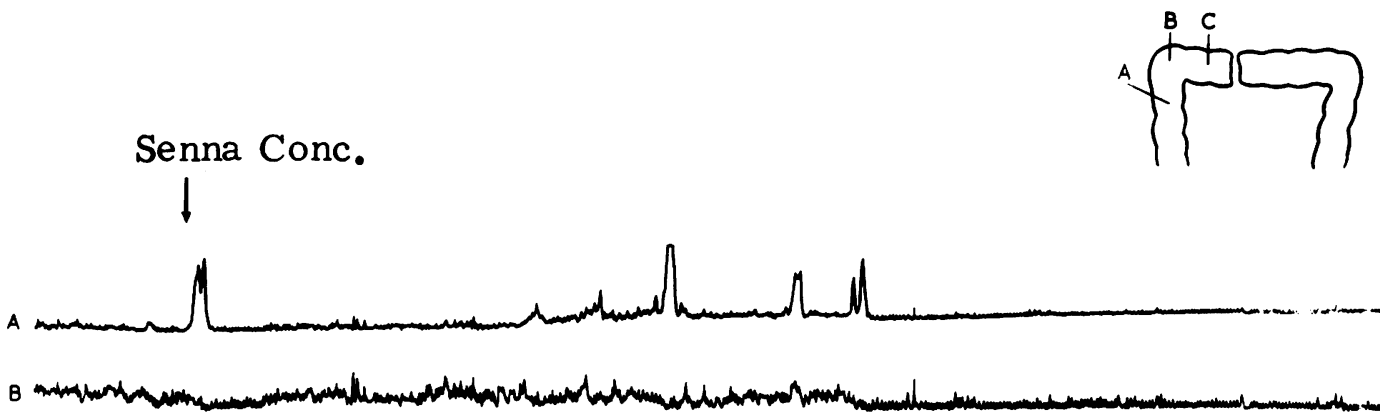

c

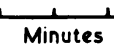

Fig. 1 Effect of $0.5 \mathrm{~g}$ of senna concentrate incubated in Brewer's_medium inserted into the transverse colon.

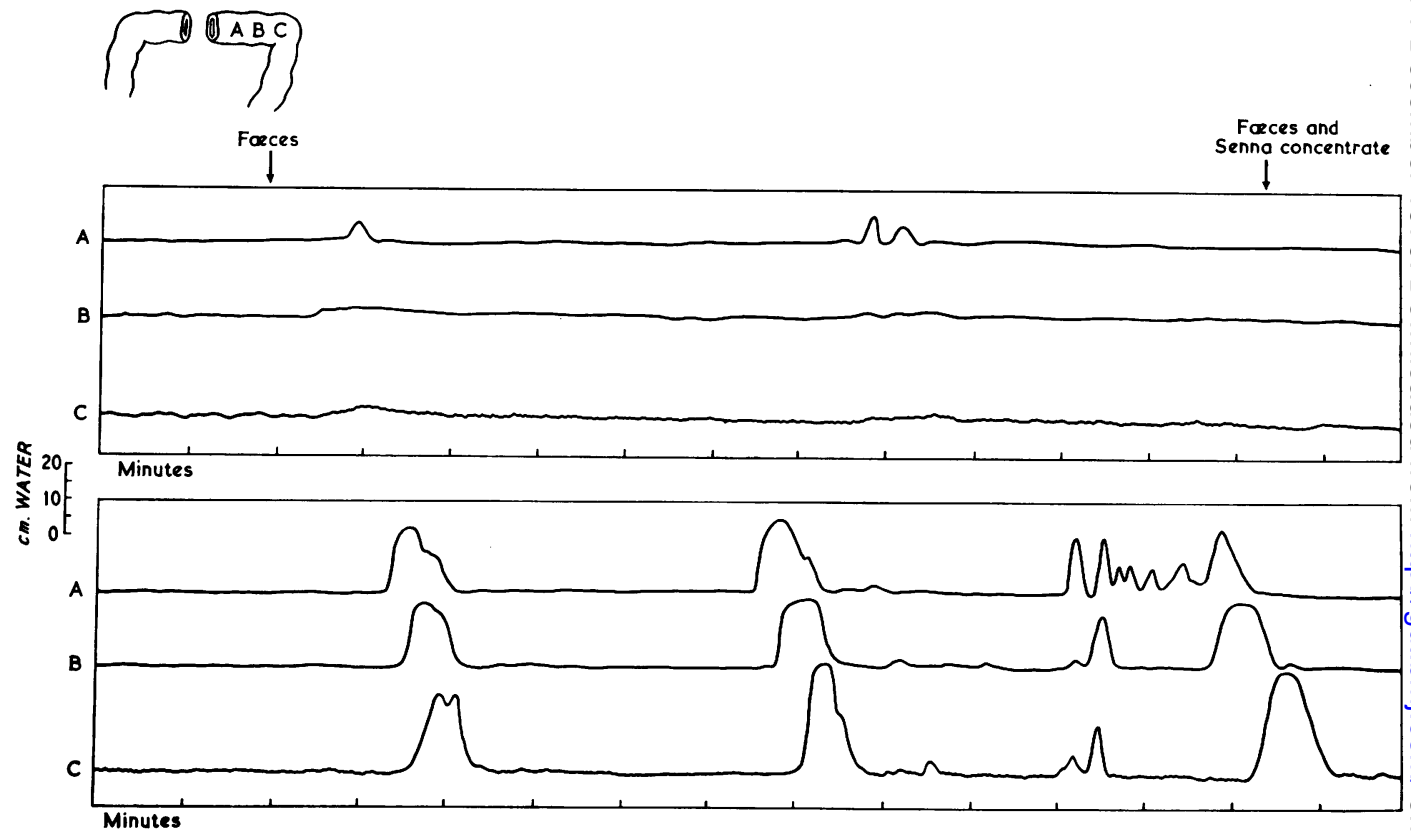

Fig. 2 Effect of faeces incubated in Brewer's medium and of faeces and $0.5 \mathrm{~g}$ senna concentrate incubated in Brewer's medium inserted into the transverse colon.

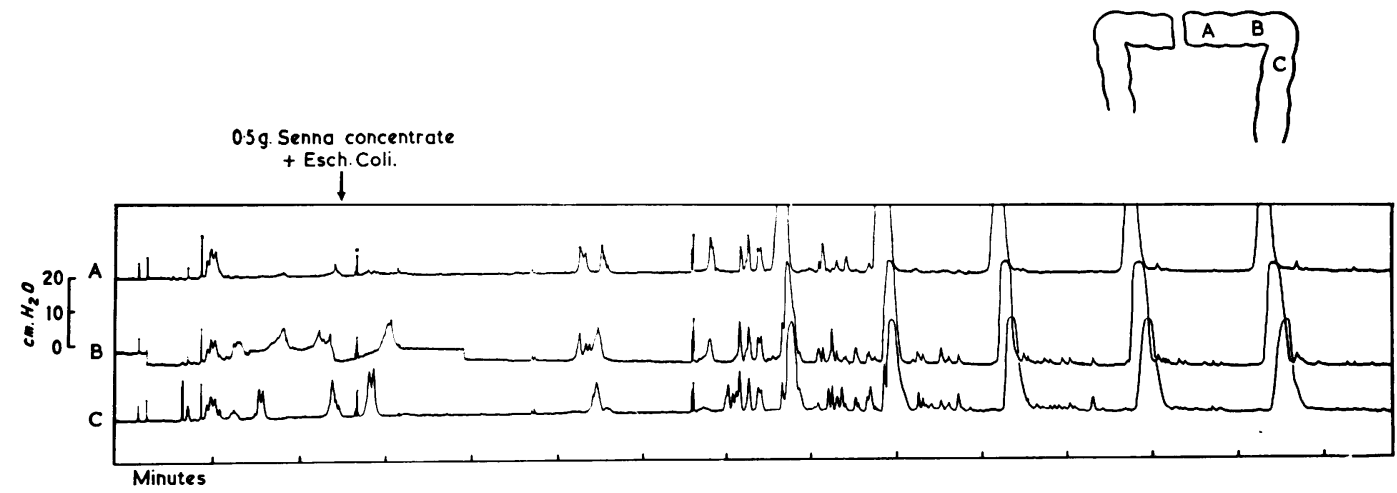

Fig. 3 Effect of $0.5 \mathrm{~g}$ senna concentrate and pure Esch. coli cultures incubated in Brewer's medium inserted into the transverse colon. 
the human colon or rectum did not stimulate peristalsis during the period studied.

After incubation with faeces for periods from six to 12 hours, the senna concentrate was changed so that it then stimulated repetitive peristaltic waves which occurred within 10 minutes of the stimulus being applied and lasted throughout the period studied. This activation was more likely to be produced if the substances were incubated in Brewer's medium which supports both aerobic and anaerobic bacterial growth.
Using a subculture of Esch. coli from the patient's faeces and then incubating with senna concentrate for three hours, a similar peristaltic response was demonstrated. These results are in agreement with animal experiments performed by Okada (1940). Thus it would appear that the glycosides contained in senna can be changed by incubation in vitro with faeces or $E$. coli into substances which directly stimulate the human colon to peristalt. It would therefore seem likely that oral senna is activated in the colon by bac-

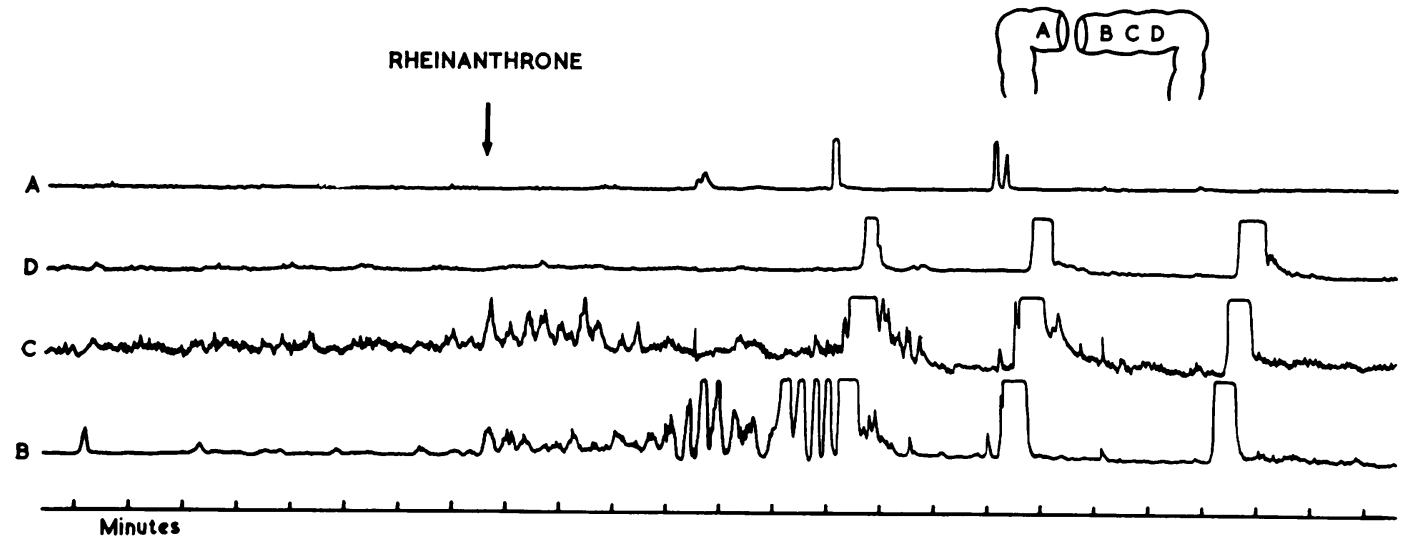

Fig. 4 Effect of rheinanthrone in Brewer's medium inserted into the transverse colon.

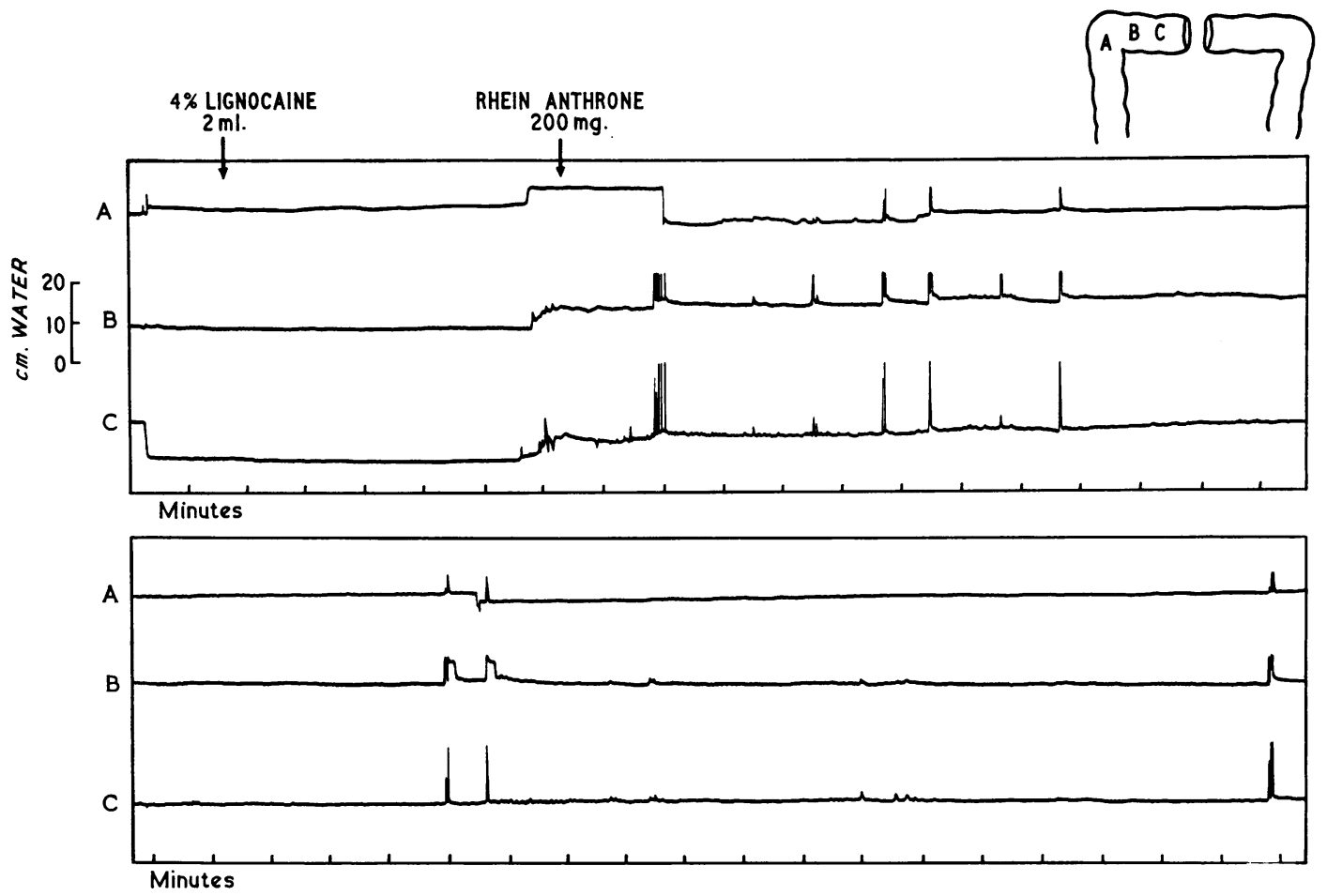

Fig. 5 Effect of surface anaesthetic ( $2 \mathrm{ml} 4 \%$ lignocaine) introduced into the colon before insertion of rheinanthrone. 


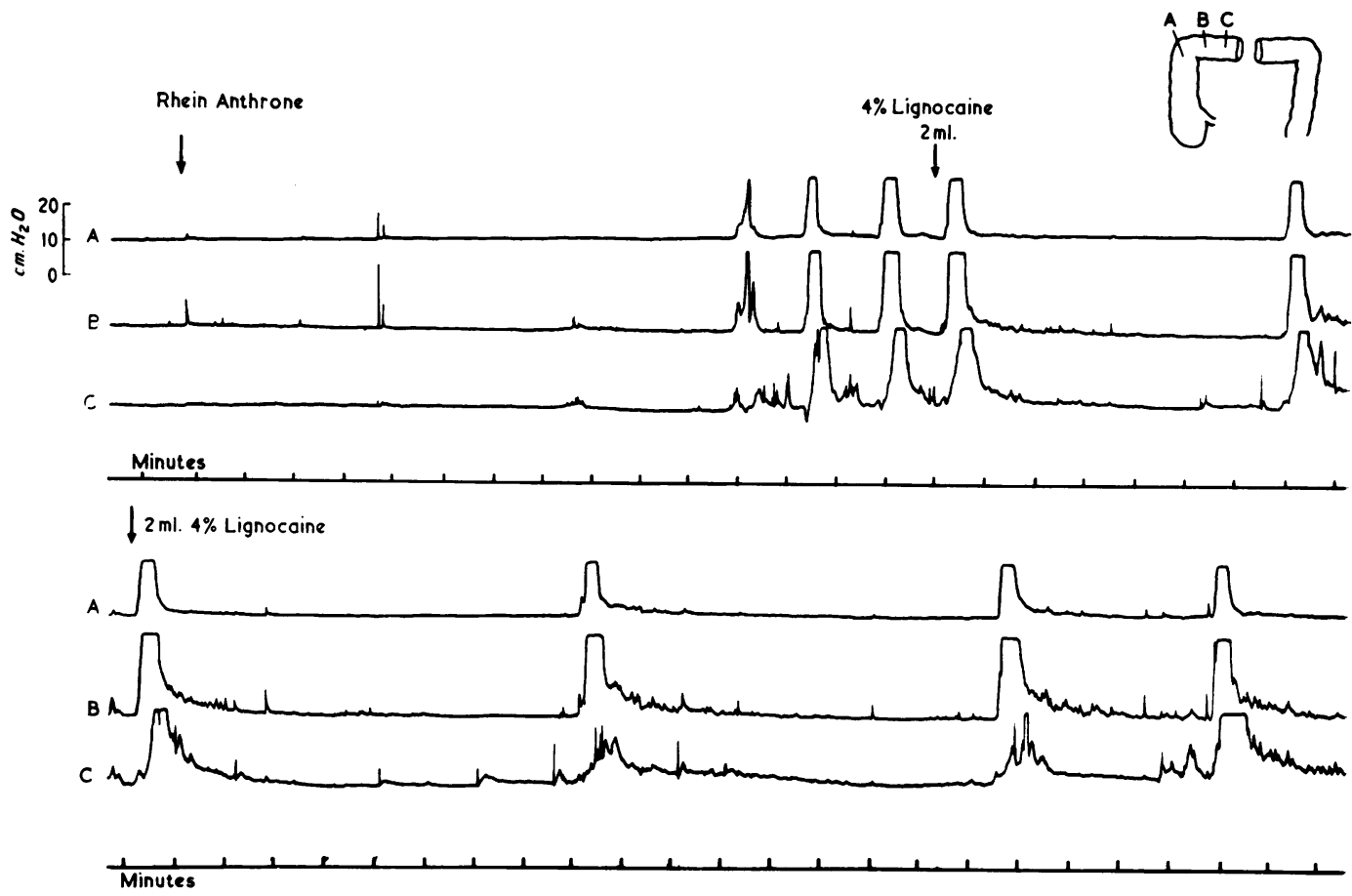

Fig. 6 Effect of surface anaesthetic ( $2 \mathrm{ml} 4 \%$ lignocaine) introduced into the colon after insertion of rheinanthrone.

terial action. The active principle is unknown but it may be similar to the oxanthrone, rheinanthrone, which can be formed by the chemical breakdown of sennosides. This may not be the only method by which sennosides are activated when taken by mouth. Chemical hydrolysis in the small bowel (Straub and Triendl, 1937) is at present being investigated.

A surface acting local anaesthetic inserted into the colon before either senna incubated with faeces or rheinanthrone blocked the response in all but one study, and this was a reduced response. If local anaesthetic was inserted after a peristaltic response had been stimulated there was either no reduction in the response or only minor alteration. It therefore seems that activated senna acts by contact stimulation of the submucosal nerve plexus which in turn excites the deeper intermuscular plexus (Hardcastle and Mann, 1968). If the submucosal plexus or mucosal receptors are blocked by local anaesthetic the response is abolished but if the intermuscular plexus has already been sensitized the peristaltic response continues even if subsequently the plexus is blocked.

In the rectum peristalsis was not observed in any patient during the 60 -minute test period as is the case with other contact stimuli (Hardcastle and Mann, 1968). In three patients, however, an increase in baseline pressure was recorded when rheinanthrone or activated senna was used]and it is therefore possible that an increase in rectal tone was stimulated.

It seems possible that senna is activated slowly after entering the colon, thereby exerting its main action in the transverse or descending colon. Peristalsis at this site is more likely to produce a formed stool. whilst a contact stimulant that is immediately active on entering the caecum may produce a more liquid stool.

\section{References}

Brewer, J. H. (1940). A clear liquid medium for the 'aerobic' cultivation of anaerobes. (Abstr.) J. Bact., 39, 10.

Fairbairn, J. W. (1949). The active constituents of the vegetable purgatives containing anthracene derivatives. I. Glycosides and aglycones. J. Pharm. Pharmacol., 1, 683-691.

Hardcastle, J. D., and Mann, C. V. (1968). Study of large bowel peristalsis. Gut, 9, 512-520.

Hardcastle, J. D., and Mann, C. V. (1970). Physical factors in the stimulation of colonic peristalsis. Gut, 11, 41-46.

Illingworth, R. S. (1953). Abnormal substances excreted in human milk. Practitioner, 171, 533-538.

Kussmaul, W., and Becker, B. (1947). Colorimetric determination of the glycosides of senna. Helv. chim. Acta, 41, 1, 28572860.

Okada, T. (1940). Über der Mechanismus der Sennaabführung. Tohoku J. exp. Med., 38, 33-42.

Schmidt, H. J. (1960). Wissenwertes für die pharmazeutische Praxis. Die Chemie und Pharmakologie Slanzlicher Laxartien. Mitt. dtsch. pharm. Ges., 30, 41-47.

Straub, W., and Triendl, E. (1937). Theoric der Abführwirkung der Folia Sennae und ihrer wirksamen Inhaltsstoffe. Naunyn-Schmiedeberg's Arch. exp. Path. Pharmak., 185, 1-19. 